\title{
The SW Sextantis-type star 2MASS J01074282+4845188: an unusual bright accretion disk with non-steady emission and a hot white dwarf ${ }^{\star}$
}

\author{
T. Khruzina ${ }^{1}$, D. Dimitrov ${ }^{2}$, and D. Kjurkchieva ${ }^{3}$ \\ 1 Moscow MV Lomonosov State University, Sternberg Astronomical Institute, 119991 Moscow, Russia \\ e-mail: kts@sai.msu.ru \\ 2 Institute of Astronomy and National Astronomical Observatory, Bulgarian Academy of Science, 1784 Sofia, Bulgaria \\ e-mail: dinko@astro.bas.bg \\ 3 Shumen university, 9700 Shumen, Bulgaria \\ e-mail: d.kyurkchieva@shu-bg.net
}

Received 19 September 2012 / Accepted 22 January 2013

\section{ABSTRACT}

\begin{abstract}
Context. Cataclysmic variables (CVs) present a short evolutional stage of binary systems. The nova-like stars are rare objects, especially those with eclipses (only several tens). But precisely these allow to determine the global parameters of their configurations and to learn more about the late stage of stellar evolution.

Aims. The light curve solution allows one to determine the global parameters of the newly discovered nova-like eclipsing star 2MASS J01074282+4845188 and to estimate the contribution of the different light sources.

Methods. We present new photometric and spectral observations of 2MASS J01074282+4845188. To obtain a light curve solution we used a model of a nova-like star whose emission sources are a white dwarf surrounded by an accretion disk, a secondary star filling its Roche lobe, a hot spot and a hot line. The obtained global parameters are compared with those of the eclipsing nova-like UX UMa. Results. 2MASS J01074282+4845188 shows the deepest permanent eclipse among the known nova-like stars. It is reproduced by covering the very bright accretion disk by the secondary component. The luminosity of the disk is much bigger than that of the rest light sources. The determined high temperature of the disk is typical for that observed during the outbursts of CVs. The primary of 2MASS J01074282+4845188 is one of the hottest white dwarfs in CVs. The temperature of $5090 \mathrm{~K}$ of its secondary is also quite high and more appropriate for a long-period SW Sex star. It might be explained by the intense heating from the hot white dwarf and the hot accretion disk of the target.

Conclusions. The high mass accretion rate $\dot{M}=8 \times 10^{-9} M_{\odot} \mathrm{yr}^{-1}$, the broad and single-peaked $\mathrm{H} \alpha$ emission profile, and the presence of an S-wave are sure signs for the SW Sex classification of 2MASS J01074282+4845188. The obtained flat temperature distribution along the disk radius as well as the deviation of the energy distribution from the black-body law are evidence of the non-steady emission of the disk. It can be attributed to the low viscosity of the disk matter due to its unusual high temperature. The close values of the disk temperature and the parameter $\alpha_{g}$ of 2MASS J01074282+4845188 and those of the cataclysmic stars at eruptions might be considered as an additional argument for the permanent active state of nova-like stars.
\end{abstract}

Key words. binaries: eclipsing - novae, cataclysmic variables - white dwarfs - accretion, accretion disks stars: individual: 2MASS J01074282+4845188

\section{Introduction}

Cataclysmic variables (CVs) consist of a white dwarf surrounded by an accretion disk and a late main sequence star filling its Roche lobe. The nova-like stars are nonmagnetic cataclysmic variables that do not truly erupt. It is assumed that they are in state of permanent eruption due to their high accretion rate. According to the standard models, the dominant emission sources of nova-like variables are the accretion disks. The second light contribution comes from the hot spot at the region where the accretion stream from the secondary star falls on the disk. These configurations explain the hump, flickering, and standstill of their light curves.

Recent gas-dynamical investigations (Bisikalo et al. 1997, 1998 , 2005, etc.) revealed that there is a place of increased energy output of nova-like stars outside the disk. This region,

\footnotetext{
* Based on data collected with telescopes at Rozhen National Astronomical Observatory.
}

called hot line, is a result of the interaction of the disk halo and the inter-component envelope with the gas stream and allows one to fit the whole light curves of the nova-like stars, including the variability of their out-of-eclipse parts (Khruzina 2001; Khruzina et al. 2003).

The cataclysmic variables present a short evolutional stage of binary systems. That is why they are rare objects, especially those with eclipses (only several tens). But precisely these allow one to determine the global parameters of their configurations and to learn more about the late stage of stellar evolution. Therefore, one should not miss the chance to investigate each cataclysmic variable with an eclipse.

2MASS J01074282+4845188 = T-And0-10518 = 1SWASP $\mathrm{J} 010744.41+484458.1$ (hereafter J0107) is a star from the list of 773 eclipsing binary (EB) systems of the TrES database (Devor et al. 2008). It has been designated as an "ambiguous EB" with coordinates $\alpha=01^{\mathrm{h}} 07^{\mathrm{m}} 44^{\mathrm{s}} .417, \delta=+48^{\circ} 44^{\prime} 58^{\prime \prime} .11$ and an orbital period of 0.1935761 . 
Table 1. Journal of the Rozhen photometric and spectral observations.

\begin{tabular}{lcccccc}
\hline \hline Date & Filter & Exp. [s] & $N$ & Phase range & Telescope & Source \\
\hline 2011 Jan. 29 & $V, I$ & 120,120 & 67,67 & whole cycle & $60-\mathrm{cm}$ & Paper I \\
2011 Jan. 30 & $V, I$ & 120,120 & 65,65 & whole cycle & $60-\mathrm{cm}$ & Paper I \\
2011 Jan. 31 & $R$ & 60 & 222 & 0.86 cycle & $60-\mathrm{cm}$ & Paper I \\
2011 Feb. 07 & spectra & 300 & 4 & 0.1 cycle & $2-\mathrm{m}$ & Paper I \\
2011 Mar. 12 & spectra & 300 & 4 & half cycle & $2-\mathrm{m}$ & new \\
2011 Aug. 18 & $R$ & 60 & 58 & half cycle & $60-\mathrm{cm}$ & new \\
2011 Nov. 04 & spectra & 300 & 2 & 0.05 cycle & $2-\mathrm{m}$ & new \\
2011 Nov. 11 & $B$ & 90 & 261 & more one cycle & $2 \mathrm{~m}$ & new \\
2011 Dec. 16 & $V$ & 60 & 262 & whole cycle & Schmidt & new \\
\hline
\end{tabular}

The recent photometric observations of Dimitrov \& Kjurkchieva (2012, hereafter Paper I) led to the conclusion that Devor et al. (2008) misidentified the variable T-And0-10518 for its neighbor, a considerably brighter star (standard star St8 in Fig. 2 and Table 1 of Paper I) at distance of $26^{\prime \prime}$ from the true variable. The misidentification is due to the low spatial resolution of the TrES observations. As a result of the impossibility to separate the contributions of the close stars, the determined initial variability amplitude has been almost 11 times smaller than the true amplitude of the fainter star.

The follow-up observations with a bigger telescope (Paper I) than the one used for the TrES survey allowed us to separate the two close stars, to determine which of them is the variable, and to obtain the correct variability amplitude of the precise light curve. It was found that the VRI light curves of the target have a very deep, asymmetric minimum. The $\mathrm{V}$-shaped light minimum, the characteristic phase dependence of the color indices $V-R$ and $V-I$, the pre-eclipse hump in the light curves, and the flickering led to the conclusion that this star belongs to the novalike CVs. The observed broad $\mathrm{H} \alpha$ emission profile confirmed the photometric classification. The detailed analysis of the photometric and spectral data of J0107 led to the suggestion that it belongs to the nova-like CVs of SW Sex subtype (Paper I).

The goal of our present study is to obtain a common light curve solution for the data from Paper I and the new photometric data and to analyze the global parameters of the newly discovered cataclysmic star J0107.

\section{Rozhen observations and initial analysis}

\subsection{Photometry}

The photometric observations of J0107 at the Rozhen National Astronomical Observatory (Table 1) were carried out with (i) the 60-cm Cassegrain telescope using the FLI PL09000 CCD camera $(3056 \times 3056$ pixels, $12 \mu \mathrm{m} /$ pixel, field of $27.0 \times$ 27.0 arcmin with focal reducer); (ii) the 50/70-cm Schmidt telescope equipped by the CCD camera FLI PL16803 $(4096 \times$ 4096 pixels, $9 \mu \mathrm{m} / \mathrm{pixel}$, field of $73.3 \times 73.3 \mathrm{arcmin}$ ); (iii) the 2-m RCC telescope with the CCD camera VersArray $512(512 \times$ 512 pixels, $24 \mu \mathrm{m} /$ pixel, field of $7.6 \times 7.6 \mathrm{arcmin})$. The average accuracy of the photometric observations in the filters $B, V, R$ and $I$ was $0.010,0.020,0.010$, and 0.008 .

The new photometric data were reduced in the same way as in Paper I and the standard stars were the same.

The new folded light curves (Figs. 1, 2) were built using the ephemeris (Paper I)

$\mathrm{HJD}(\operatorname{Min})=2454417.382244(25)+0.1935980(11) \times \mathrm{E}$.
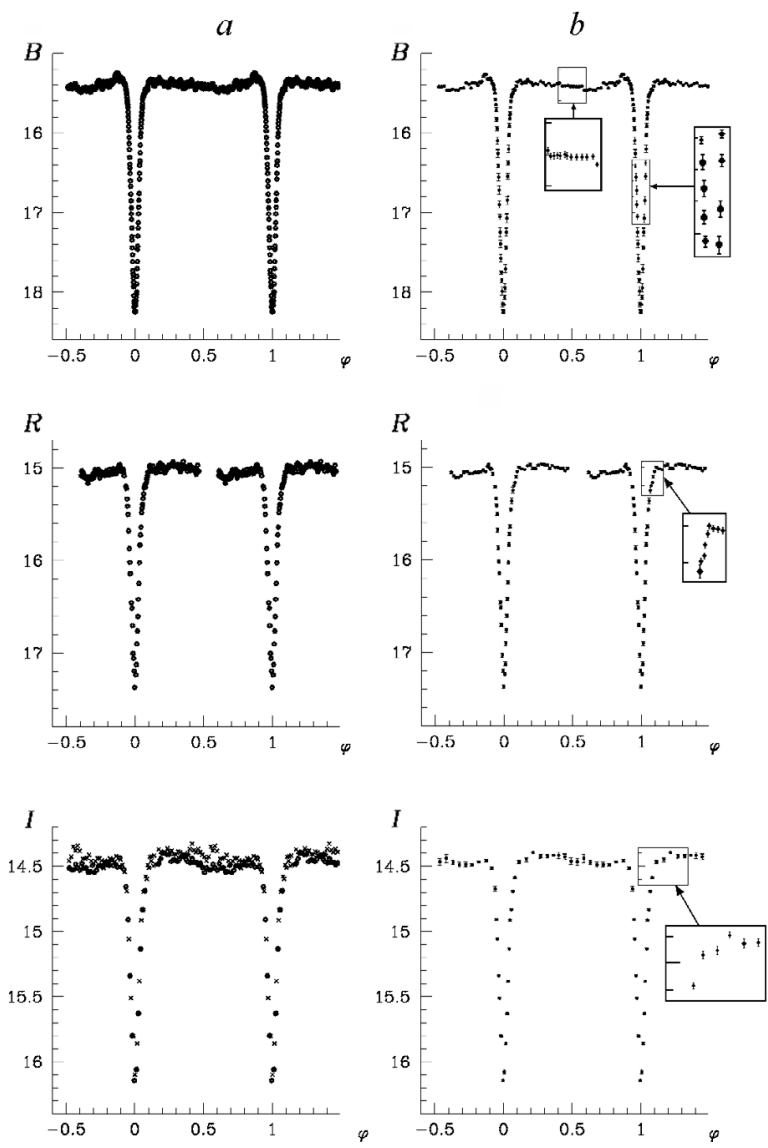

Fig. 1. Individual (a) and average (b) BRI light curves of J0107. The $I$ photometric data are shown by different symbols: points for 2011 Jan. 29 and crosses for 2011 Jan. 30. The marked parts of the light curves are also shown on a large scale for better visibility of the error bars.

The qualitative analysis of all photometric data led to several conclusions.

(i) The out-of-eclipse light level changes from one orbital cycle to another. Figure 1 illustrates this with the data in filter $I$ obtained in two consecutive nights in January 2011. A similar behavior is visible in Fig. 2 of Paper I for the $V$ data.

(ii) The brightness of J0107 changes on time scales of months. We found that the light levels during December 2011 both into and out of the eclipse are lower by around $\Delta V \sim$ $0.10-0$. 15 than those obtained in January 2011, while the variability amplitudes are the same (Fig. 2). The brightness of both curves, $V_{1}$ from January and $V_{2}$ from December 2011, is the same only at phases $\varphi \sim 0.10-0.15$. 

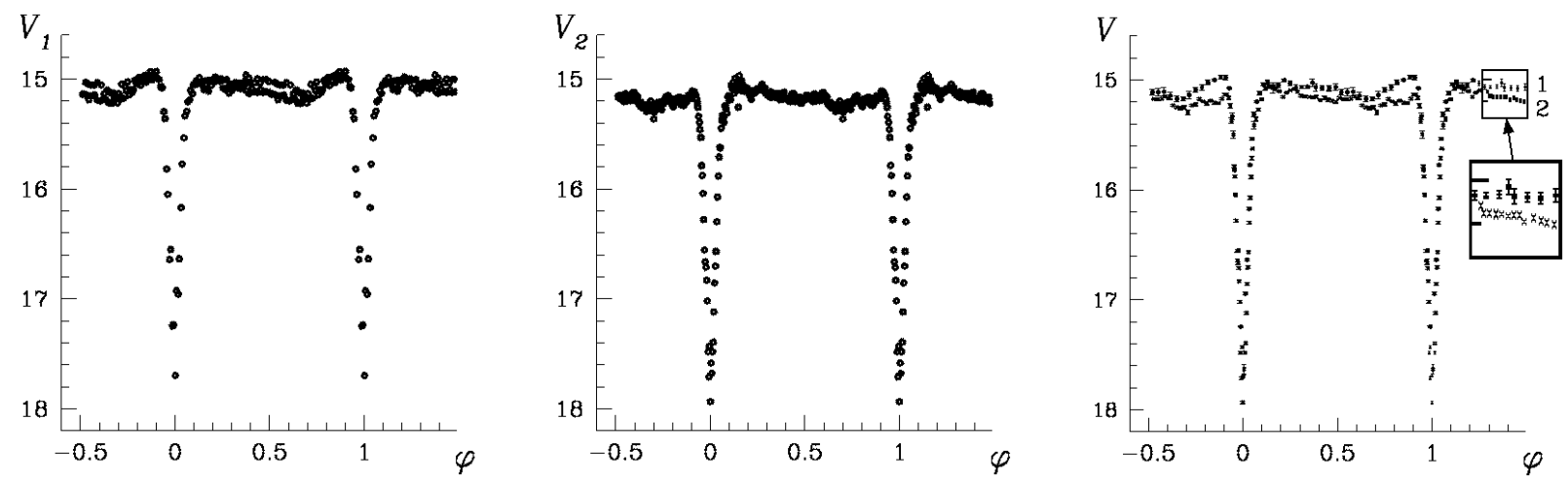

Fig. 2. From left to right: light curve $V_{1}$ of $\mathrm{J} 0107$ from January 2011; light curve $V_{2}$ from December 2011; average $V$ light curve (the symbols 1 and 2 are for curves $V_{1}$ and $V_{2}$ ). The marked part of the light curves is also shown on a large scale for better visibility of the error bars.

Table 2. Depths and widths of the eclipse.

\begin{tabular}{ccc}
\hline \hline Filter & Depth & Width \\
\hline$B$ & 3.0 & 0.060 \\
$V$ & 2.9 & 0.062 \\
$R$ & 2.4 & 0.063 \\
$I$ & 1.7 & 0.078 \\
\hline
\end{tabular}

(iii) The pre-eclipse hump is no permanent feature of the light curve of J0107: it is clearly visible in light curve $V_{1}$ but is lacking in curve $V_{2}$. As a result, the light level after the eclipse is higher than before it in the curve $V_{2}$ (Fig. 2).

The measured depths (in mag) and widths (as a relative part of the cycle) of the eclipse minimum in different filters (Table 2) imply a higher light contribution of the accretion disk of J0107 at shorter wavelengths as well as a bigger size of the secondary at longer wavelengths.

The most distinguishable feature of J0107 is its deep eclipse (2.9 mag in $V$ ). The eclipse depths of the majority of the eclipsing nova-like variables are $\leq 1 \mathrm{mag}$. The deepest eclipses belong to the SW Sex stars. Only nine SW Sex stars in the list of Rodríguez-Gil et al. (2007) have eclipse depths above 2.0 mag, which are, however, lower than that of J0107. Two SW Sex stars, DW UMa (Stanishev et al. 2004) and V1315 Aql (Papadaki et al. 2009), have shown deeper eclipses of 3.2-3.4 mag, but only once and only within 2-3 days, whereas normally their eclipse depths are below 2.0 mag. Therefore, 2MASS J01074282+4845188 has the deepest permanent eclipse (at least during 11 months) among the known eclipsing nova-like variables.

\subsection{Spectral observations}

The spectra of the target (Table 1) were obtained with the 2-m RCC telescope that is equipped with a VersArray 512 CCD camera, a focal reducer FoReRo-2, and a grism with 300 lines/mm. The resolution of the spectra is $5.2 \AA /$ pixel and they cover the range 5000-7000 A.

The variability of the $\mathrm{H} \alpha$ emission of $\mathrm{J} 0107$ is apparent in the published four spectra of J0107 (Fig. 3 of Paper I) as well as in the six new spectra (Fig. 3 and Table 3 ).

The qualitative analysis of the spectra led to several conclusions:

(a) The intensity of the $\mathrm{H} \alpha$ emission increases from the beginning to the end of 2011 (Table 3).

(b) There is a trend of the $\mathrm{H} \alpha$ emission to be considerably higher around phase 0.75 .

(c) The $\mathrm{H} \alpha$ emission line has predominantly one-peaked profile.

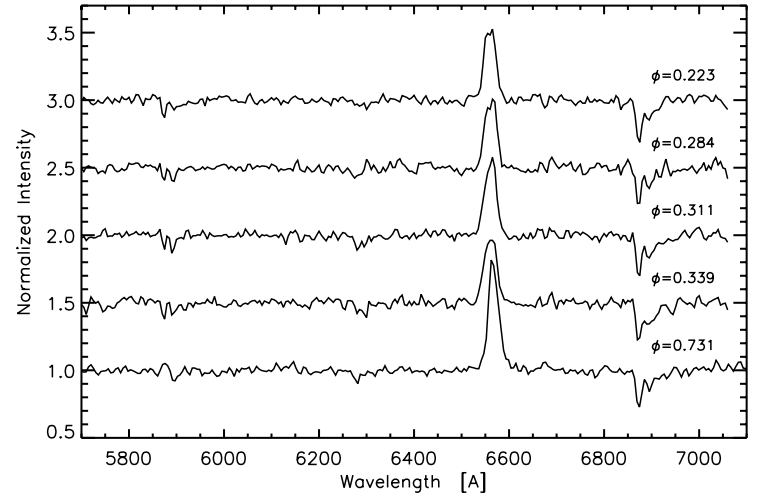

Fig. 3. New low-resolution spectra of J0107.

(d) Even in the insufficient phase coverage of our spectral observations, the $\mathrm{H} \alpha$ line reveals an S-wave with an amplitude of at least $270 \mathrm{~km} \mathrm{~s}^{-1}$ (Table 3). This is one of the most important criteria for an SW Sex membership.

(e) The high values of the full width at zero intensity (FWZI) of the $\mathrm{H} \alpha$ line (Table 3) are typical of SW Sex stars (Thorstensen et al. 1991).

\section{Light curve solution of the Rozhen photometry of $\mathrm{J} 0107$}

\subsection{Model for the light curve solution}

To determine the parameters of the newly discovered CV J0107 we used the model of Khruzina (2011, hereafter Model TK) which is based on several assumptions.

1. It takes into account the effects of reflection, gravity darkening, and linear limb darkening of the two stellar components: the spherical white dwarf and the late secondary filling its Roche-lobe, whose separation $a_{0}$ is assumed to be a distance unit $\left(a_{0}=1.0\right)$.

2. The accretion disk around the white dwarf is slightly elliptical $(e \leq 0.1)$ and one of its foci coincides with the white dwarf center (Fig. 4). The equatorial plane of the disk lies in the orbital plane of the system (see detailed description in Khruzina 2000).

The disk is heated mainly by the gravitational energy released by the accretion of matter on the white dwarf surface. As a result, the temperature distribution through the disk is

$T(r)=T_{\mathrm{in}}\left(\frac{R_{\mathrm{in}}}{r}\right)^{\alpha_{g}}$, 
Table 3. Parameters of the $\mathrm{H} \alpha$ line of J0107.

\begin{tabular}{lcccccc}
\hline \hline Date & Phase & $\begin{array}{c}\lambda_{c} \\
{[\AA]}\end{array}$ & $\begin{array}{c}F W H M \\
{[\AA]}\end{array}$ & $\begin{array}{c}F W Z I \\
{[\AA]}\end{array}$ & $\begin{array}{c}E W \\
{[\AA]}\end{array}$ & Intensity \\
\hline 2011 Feb. 7 & 0.759 & 6566.2 & 24.9 & 68.0 & 13.8 & 1.55 \\
2011 Feb. 7 & 0.783 & 6566.3 & 23.7 & 57.5 & 11.8 & 1.47 \\
2011 Feb. 7 & 0.842 & 6565.4 & 26.8 & 73.2 & 11.4 & 1.43 \\
2011 Feb. 7 & 0.860 & 6567.8 & 33.5 & 83.7 & 13.8 & 1.43 \\
2011 Mar. 12 & 0.223 & 6559.6 & 28.3 & 72.7 & 16.7 & 1.51 \\
2011 Mar. 12 & 0.284 & 6561.6 & 26.6 & 74.1 & 15.3 & 1.50 \\
2011 Mar. 12 & 0.311 & 6561.0 & 28.2 & 79.4 & 16.9 & 1.55 \\
2011 Mar. 12 & 0.339 & 6560.5 & 30.7 & 84.6 & 15.8 & 1.47 \\
2011 Nov. 4 & 0.731 & 6567.1 & 24.7 & 76.8 & 20.2 & 1.74 \\
2011 Nov. 4 & 0.749 & 6566.3 & 23.6 & 79.4 & 23.4 & 1.86 \\
\hline
\end{tabular}

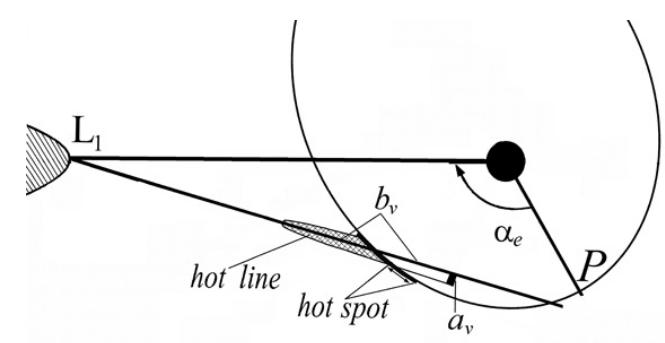

Fig. 4. Configuration of a nova-like variable: the orbital plane with the disk periastron $P$ and the optically opaque part of the flow from the secondary (shaded area).

where $R_{\text {in }}$ is the radius of the boundary layer of the disk (usually it is assumed $R_{\mathrm{in}} \sim R_{\mathrm{wd}}$ ) while $r$ is the distance from the center of the current differential disk area to the center of the white dwarf. The range of the parameter $\alpha_{g}$ is $[0.10,0.75]$ : for low values of $\alpha_{g}$ the disk is bright, while for high values of $\alpha_{g}$ the disk emission is faint. The equilibrium state of the disk corresponds to the highest value $\alpha_{q}=0.75$ (Shakura \& Sunyaev 1973).

3. The hot spot is heated by the shock wave arising from the collision of the gas stream with the rotating disk (Bisikalo et al. 1997, 1998, 2003, 2005). The geometric thickness of the disk is higher there (Fig. 5). The shape of the hot spot is described by a half ellipse on the lateral disk surface.

4. The opaque portion of the gas stream is reproduced by the hot line. This feature is also heated by the shock wave and its shape is assumed to be an elongated ellipsoid (Figs. 4, 5). The emission from the hot spot and the hot line can explain the pre-eclipse hump in the light curves of the nova-like variables. Moreover, the hot line can cause a hump at the end of the eclipse for certain orientations of its biggest axis.

5. To calculate the emission from the secondary non-spherical star we took into account three effects.

The temperature distribution due to the gravity darkening is

$T\left(r_{\mathrm{i}}\right)=T_{2}\left[\frac{g\left(r_{\mathrm{i}}\right)}{\langle g\rangle}\right]^{\beta}$,

where $g\left(r_{\mathrm{i}}\right)$ is the gravitational acceleration at a current point at distance $r_{\mathrm{i}}$ from the mass center of the secondary, and $T_{2}$ is its temperature without heating.

The heating of the secondary from the high-temperature sources of J0107 was calculated with the formula describing the heating of a star from nearby hot (X-ray) component (Balog et al. 1981). For CVs this formula is reduced to

$$
T_{\mathrm{h}}^{4}\left(r_{\mathrm{i}}\right)=T^{4}\left(r_{\mathrm{i}}\right)+L_{*} \frac{\varepsilon \cos \alpha}{4 \pi \sigma \rho^{2}}, \quad L_{*}=4 \pi \sigma T_{*}^{4} R_{\mathrm{wd}}^{2},
$$

where $\varepsilon$ is the coefficient of processing of the hightemperature emission $(\varepsilon \sim 0.5$ for hot stars and $\varepsilon \sim 1.0$ for cool stars, Basko \& Sunyaev 1973); $\alpha$ is the angle between the normal to the elementary heated area $\mathrm{d} S$ and the direction to the high-temperature source; $\rho$ is the distance between the high-temperature source and the heated area $\mathrm{d} S$. The Model TK checks the visibility of the white dwarf from each elementary area $\mathrm{d} S$ of the secondary star and then calculates its temperature according to Eq. (4). For cataclysmic stars $T_{*}$ corresponds to the temperature of the boundary layer of the accretion disk because usually it is higher than $T_{\mathrm{wd}}$ (this is amply fulfilled for our target, see below).

The limb-darkening is taken into account to calculate the emission fluxes of the secondary

$$
\begin{aligned}
F(\varphi)= & \sum_{\cos \gamma>0} B_{\lambda}\left(T_{\mathrm{h}}\left(r_{\mathrm{i}}\right)\right) \\
& \times[1-u(\lambda, T)+u(\lambda, T) \cos \gamma] \cos \gamma \mathrm{d} S,
\end{aligned}
$$

where $u(\lambda, T)$ is the limb-darkening coefficient and $B_{\lambda}\left(T_{\mathrm{h}}\left(r_{\mathrm{i}}\right)\right)$ is the Planck function corresponding to the mean wavelength of the filter.

The fitted parameters of Model TK are

(a) the mass ratio $q=M_{\mathrm{wd}} / M_{2}$ and orbital inclination $i$;

(b) the secondary temperature $T_{2}$;

(c) the radius $R_{\mathrm{wd}}$ and temperature $T_{\mathrm{wd}}$ of the white dwarf;

(d) the parameters of the accretion disk: eccentricity $e$, radius at the apoastron $R_{\mathrm{d}}$, azimuth of the disk periastron $\alpha_{\mathrm{e}}$, temperature of the boundary layer $T_{\mathrm{in}}$, temperature of the disk edge $T_{\text {out }}$, thickness of the disk outer edge (the height $z / 2$ of the disk edge above the orbital plane in terms of $a_{0}$ or the full angular thickness $\beta_{\mathrm{d}}$ of the disk edge in degrees), parameter $A$ of the paraboloid describing the internal disk surface, parameter $a$ of the ellipsoid describing the external disk surface, parameter $\alpha_{g}$ determining the temperature distribution across the disk;

(e) the parameters of the hot line: axis sizes $a_{\mathrm{v}}, b_{\mathrm{v}}$, and $c_{\mathrm{v}}$, temperature parameters $T_{\mathrm{w}}$ (windward temperature) and $T_{1}$ (leeward temperature);

(f) the radius of the hot spot $R_{\mathrm{sp}}$ in units of $a_{0}$ (the code calculates also the angle $\varphi_{\mathrm{sp}}$ (in degrees) between the axis of the hot line and the line connecting the stellar components which depends on the parameters of the disk and the hot line). 


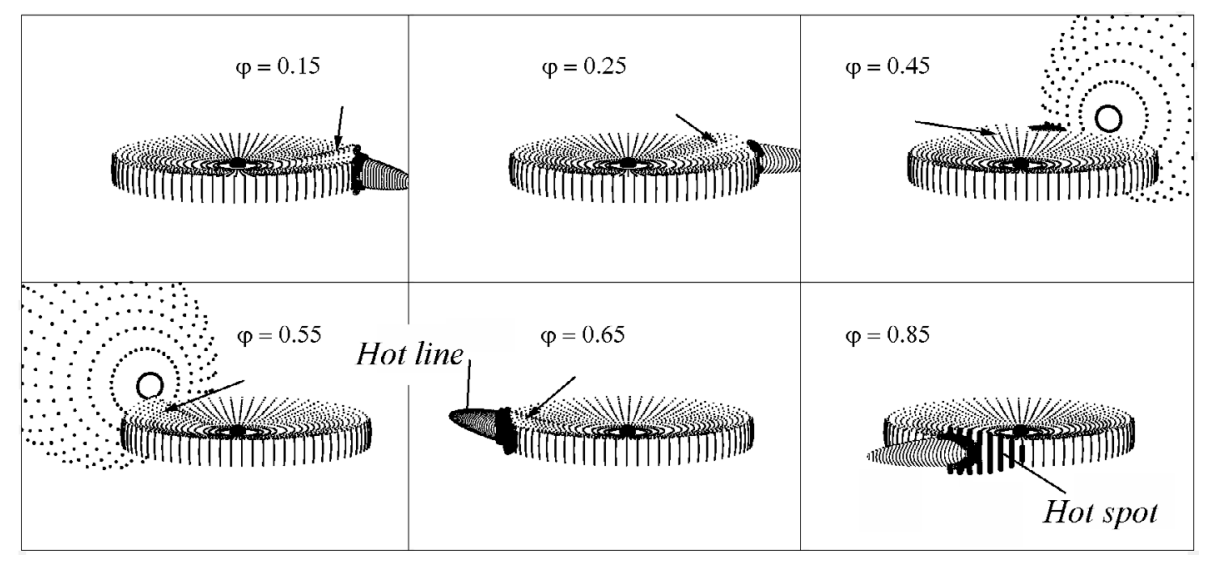

Fig. 5. 3D configurations of a nova-like variable at different orbital phases $\varphi$. The arrows show the disturbed area of the inner disk surface while the thick dots indicate the heated part of the hot line near its base.

Table 4. Parameters of variable stars of SW Sex subtype.

\begin{tabular}{lcccccc}
\hline \hline Name & $P_{\text {orb }}[$ days] & Sp type & $q=M_{\text {wd }} / M_{2}$ & $i\left[^{\circ}\right]$ & $M_{\text {wd }} / M_{\odot}$ & $M_{2} / M_{\odot}$ \\
\hline SW Sex & 0.134938 & & 1.8 & 79 & 0.58 & 0.33 \\
BT Mon & 0.333814 & G8V & 1.19 & 82 & 1.04 & 0.87 \\
V363 Aur & 0.321242 & G5-9V & 0.85 & 60 & 0.9 & 1.06 \\
& & & 1.12 & 70 & 0.86 & 0.77 \\
AC Cnc & 0.300478 & K1-3V & 0.98 & 75 & 0.76 & 0.77 \\
V347 Pup & 0.231936 & M0.5V & $1.2-2.2$ & $84-87$ & $0.63-1.2$ & $0.52-0.55$ \\
RW Tri & 0.231883 & M0V & 1.3 & 70 & 0.55 & 0.35 \\
& & & 0.76 & 79 & 0.44 & 0.58 \\
V1776 Cyg & 0.164739 & & 1.6 & 75 & 0.6 & 0.37 \\
UU Aq & 0.163805 & K7-M0V & $2.4-3.3$ & $71-83$ & $0.67-0.9$ & $0.2-0.4$ \\
LX Ser & 0.158432 & M2V & $1.14-5$ & $75-90$ & 0.41 & 0.36 \\
V380 Oph & 0.154107 & & 1.6 & 42 & 0.58 & 0.36 \\
VZ Scl & 0.144622 & & 0.7 & 90 & 1 & 1.4 \\
V1315 Aql & 0.139690 & & 2.9 & 82 & 0.73 & 0.30 \\
DW UMa & 0.136607 & M3-9 & 2.6 & 82 & 0.77 & 0.30 \\
J0809+3814 & 0.134038 & & 3.3 & 65 & 1.0 & 0.3 \\
V348 Pup & 0.101839 & & 3.2 & 81 & 0.65 & 0.20 \\
EX Hya & 0.068234 & M5-6V & $4.4-7.9$ & 78 & $0.7-1.5$ & $0.1-0.2$ \\
\hline
\end{tabular}

\subsection{Preliminary considerations and procedure of the light curve solution}

We present several considerations that allow us to narrow some ranges of the unknown model parameters.

(a) To decrease the range of the mass ratio we assumed that J0107 belongs to the subtype SW Sex of the nova-like variables (Paper I). According to the catalogs of Ritter \& Kolb (2003) and Cherepashchuk et al. (1996), the mass ratios of the SW Sex systems are in the range $q \sim 0.8-7.9$ with a peak near $q \sim 1-3$ (Table 4 ).

We were unable to use the eclipse width as an estimate of the mass ratio $q=M_{\mathrm{wd}} / M_{2}$ and orbital inclination $i$ of the system (Littlefair et al. 2008) because the time resolution of the Rozhen photometric observations of J0107 is insufficient.

(b) For the standard configuration of a nova-like variable the disk radius is about half of the Roche lobe and its eclipse (at least partial) is possible for orbital inclinations $i>60^{\circ}$. Despite the deep eclipse of J0107 we assumed the initial condition $i>40^{\circ}$.

(c) The spectral types of the secondary components of the SW Sex stars are G-M (Table 4). Therefore we varied the secondary temperature in the wide range $T_{2} \sim$ $3000-6000 \mathrm{~K}$.

Our spectra of J0107 do not allow us to constraint the secondary temperature (it is too weak to show some features in our low-resolution spectra). It is not reasonable either to use the empirical relationship of Warner (1995) between the orbital periods of CVs and the absolute visual magnitudes of their secondaries because it is based on only five nova-like stars.

For the remaining fitted parameters we used the standard ranges: $R_{\mathrm{d}} / \xi \sim 0.3-0.9$ ( $\xi$ is the distance between the mass center of the white dwarf and $L_{1}$ whose value depends on $q$ and is calculated with the code); $A \sim 3-7 ; \alpha_{\mathrm{e}} \sim 0-90^{\circ} ; T_{\text {in }}=\gamma T_{\text {wd }}$ where $\gamma \sim 1-7$ (the temperature of the boundary layer $T_{\text {in }}$ determines the temperature distribution along the disk radius, the heating of the secondary component and the heating of the inner disk regions near the outer edge); $\alpha_{g} \sim 0.1-0.75 ; R_{\mathrm{wd}} / \xi \sim$ $0.001-0.05$ or $R_{\mathrm{wd}} \sim(0.001-0.03) a_{0} ; T_{\mathrm{wd}} \sim 10000-70000 \mathrm{~K}$; $T_{\mathrm{l}}, T_{\mathrm{w}} \sim 200-90000 \mathrm{~K} ; R_{\mathrm{sp}} / R_{\mathrm{d}} \sim 0-0.25 ; a_{\mathrm{v}} \sim(0.1-0.4) R_{\mathrm{d}}$, $b_{\mathrm{v}} \sim(0.2-0.9) R_{\mathrm{d}}, c_{\mathrm{v}} \sim(1-1.8) z$.

To fit our data we used the code based on the Model TK. It calculates the light fluxes using the Planck energy distribution for the corresponding central wavelength $\lambda_{0}$ of the filters: $4400 \AA$ for filter $B, 5500 \AA$ for filter $V, 6960 \AA$ for filter $R$, and $8800 \AA$ for filter $I$ (Johnson 1965). For the transfer from synthetic light fluxes to magnitudes we used the value $F_{V}=1.66$ in conventional units corresponding to $V=15$. 09 . Using this conventional unit is necessary because the Planck function defines the energy flux through the unit area $\left(1 \mathrm{~cm}^{2}\right)$ while the code works with a distance unit $a_{0}$ whose value is unknown in advance. 
Table 5. Parameters of the light curve solution of J0107.

\begin{tabular}{|c|c|c|c|c|c|c|}
\hline Parameters & Units & $B$ & $V_{1}$ & $V_{2}$ & $R$ & $I$ \\
\hline$n$ & & 114 & 43 & 75 & 71 & 35 \\
\hline$q=\frac{M_{\mathrm{wd}}}{M_{2}}$ & & & & $2.332 \pm 0.023$ & & \\
\hline & $\circ$ & & & $81.4 \pm 0.1$ & & \\
\hline$T_{2}$ & $\mathrm{~K}$ & & & $5090 \pm 20$ & & \\
\hline$\left\langle R_{2}\right\rangle$ & $a_{0}$ & & & $0.318 \pm 0.001$ & & \\
\hline$\xi$ & $a_{0}$ & & & $0.586 \pm 0.001$ & & \\
\hline & & & & White dwarf & & \\
\hline$R_{\mathrm{wd}}$ & $\xi$ & & & $0.0113 \pm 0.0011$ & & \\
\hline$R_{\mathrm{wd}}$ & $a_{0}$ & & & $0.0066 \pm 0.0006$ & & \\
\hline$T_{\mathrm{wd}}$ & $\mathrm{K}$ & & & $40000 \pm 600$ & & \\
\hline & & & & Accretion disk & & \\
\hline$R_{\mathrm{d}}$ & $\xi$ & $0.362 \pm 0.009$ & $0.46 \pm 0.03$ & $0.381 \pm 0.005$ & $0.462 \pm 0.003$ & $0.50 \pm 0.04$ \\
\hline$a$ & $a_{0}$ & $0.211 \pm 0.005$ & $0.27 \pm 0.02$ & $0.223 \pm 0.003$ & $0.270 \pm 0.001$ & $0.29 \pm 0.02$ \\
\hline$A$ & & $4.6 \pm 0.3$ & $4.6 \pm 0.9$ & $4.5 \pm 0.1$ & $4.60 \pm 0.03$ & $4.6 \pm 0.8$ \\
\hline$z / 2$ & $a_{0}$ & $0.0100 \pm 0.0002$ & $0.013 \pm 0.006$ & $0.010 \pm 0.001$ & $0.013 \pm 0.001$ & $0.014 \pm 0.001$ \\
\hline$\beta$ & $\circ$ & $5.38 \pm 0.02$ & $5.4 \pm 0.9$ & $5.64 \pm 0.02$ & $5.43 \pm 0.02$ & $5.42 \pm 0.02$ \\
\hline$T_{\text {in }}$ & $\mathrm{K}$ & $44110 \pm 820$ & $52470 \pm 7200$ & $52350 \pm 800$ & $58780 \pm 13100$ & $58900 \pm 9550$ \\
\hline$T_{\text {out }}$ & $\mathrm{K}$ & $17300 \pm 110$ & $23800 \pm 300$ & $28600 \pm 110$ & $31350 \pm 75$ & $32150 \pm 140$ \\
\hline \multirow[t]{2}{*}{$\alpha_{g}$} & & $0.27 \pm 0.04$ & $0.22 \pm 0.04$ & $0.173 \pm 0.005$ & $0.17 \pm 0.01$ & $0.16 \pm 0.04$ \\
\hline & & \multicolumn{5}{|c|}{ Hot line and hot spot } \\
\hline$a_{v}$ & $a_{0}$ & $0.02 \pm 0.01$ & $0.09 \pm 0.04$ & $0.08 \pm 0.01$ & $0.13 \pm 0.01$ & $0.04 \pm 0.02$ \\
\hline$b_{v}$ & $a_{0}$ & $0.213 \pm 0.008$ & $0.30 \pm 0.03$ & $0.26 \pm 0.01$ & $0.36 \pm 0.01$ & $0.24 \pm 0.02$ \\
\hline$c_{v}$ & $a_{0}$ & $0.010 \pm 0.001$ & $0.02 \pm 0.01$ & $0.019 \pm 0.002$ & $0.023 \pm 0.001$ & $0.017 \pm 0.002$ \\
\hline$T_{w}$ & $\mathrm{~K}$ & $50000 \pm 2060$ & $77500 \pm 3770$ & $85120 \pm 3700$ & $67640 \pm 1700$ & $57110 \pm 2540$ \\
\hline$T_{l}$ & $\mathrm{~K}$ & $22750 \pm 3900$ & $1710 \pm 240$ & $1850 \pm 350$ & $2510 \pm 1600$ & $27180 \pm 2330$ \\
\hline$R_{\mathrm{sp}}$ & $a_{0}$ & $0.10 \pm 0.02$ & $0.2 \pm 0.1$ & $0.13 \pm 0.07$ & $0.24 \pm 0.02$ & $0.13 \pm 0.04$ \\
\hline$\varphi_{\mathrm{sp}}$ & & $8.5 \pm 0.5$ & $9 \pm 2$ & $6 \pm 2$ & $6.3 \pm 0.1$ & $14 \pm 4$ \\
\hline$\chi^{2}$ & & 9177 & 3419 & 4607 & 3148 & 1774 \\
\hline$\chi_{0,001, n}^{2}$ & & 168.5 & 78.2 & 120.1 & 115 & 66.9 \\
\hline
\end{tabular}

We used the tables of Al-Naimiy (1978) and Grygar et al. (1972) for the values of the stellar limb-darkening coefficients $u(\lambda, T)$ corresponding to the wavelengths of the filters and stellar temperatures and the value $u_{\mathrm{d}}=2 / 3$ for the accretion disk (Shakura \& Sunyaev 1973).

To search for the best fit the code uses the method of Nelder-Mead (Himmelblau 1972). An estimate of the fitting quality of the theoretical and observed light curves is the sum of the residuals

$\chi^{2}=\sum_{j=1}^{n} \frac{\left(m_{j}^{\text {theor }}-m_{j}^{\text {obs }}\right)^{2}}{\sigma_{j}^{2}}$,

where $m_{j}^{\text {theor }}$ and $m_{j}^{\text {obs }}$ are the theoretical and observational magnitudes at the $j$ th orbital phase, $\sigma_{j}$ is the dispersion of the $j$ th point and $n$ is the number of the points on the light curve.

It is known that an inverse problem solution is a combination of model parameters for which the sum of the residuals $\chi^{2}$ given by Eq. (6) is below the corresponding critical level of $\chi_{\eta, n}^{2}$ (whose tabulated values increase both with increasing $n$ and decreasing $\eta$ ). Usually, the value of the probability $\eta$ to reject the correct solution is chosen to be 0.001 .

But there are cases for which the values of $\chi^{2}$ of all solutions are above the critical level of $\chi_{\eta, n}^{2}$. These situations may arise from the small errors $\sigma_{i}$ of the normal points of the observed light curves (see Eq. (6)) and/or from some imperfections of the model (neglecting of some effects). Then it is impossible to determine the "true" uncertainties of the model parameters. In these cases it is appropriate to estimate the stability of the solution, i.e., the impact of changing a given parameter on the solution quality (Cherepashchuk 1993; Abubekerov et al. 2008).
For this aim it is necessary to introduce the "conditional" sum of the residuals $\chi^{2}(+N \%)$. Its value is higher by $N \%$ than the sum of the residuals of the considered solution (usually $N \%=10 \%$ ).

In order to find the global minimum of the residuals in the parameter space we carried out the calculations in two stages.

Firstly, we searched for solutions for each filter separately and for the whole considered ranges of the model parameters. Since the dependence of the light curve shape on the mass ratio is weak, this parameter was varied by step of $\Delta q=0.1$. As a result we determined the intersections of the ranges of the main system parameters obtained for the different filters: $q=[2.33-2.40] ; i=$ $[81-83]^{\circ} ; T_{2}=[4700-5200] \mathrm{K} ; R_{\mathrm{wd}} / \xi=[0.01130-0.01145] ;$ $T_{\text {wd }}=[38000-41000] \mathrm{K} ; A=[4.59-4.61]$. By subsequently varying the main model parameters in these narrower ranges we obtained the value: $q=2.332 ; i=81.4^{\circ} ; R_{\mathrm{wd}}=0.0114 \xi ; T_{\mathrm{wd}}=$ $40000 \mathrm{~K} ; T_{2}=5090 \mathrm{~K}$.

At the second stage we fixed the values of the main model parameters and varied the remaining model parameters that describe the disk, the hot line, and the spot.

Table 5 presents the results of the final light curve solution. The average synthetic light curves corresponding to the parameters from Table 5 are shown in Figs. 6, and 7.

The uncertainties of the main parameters of the system $q, i$, $T_{2}, T_{\mathrm{wd}}, R_{\mathrm{wd}}$ given in Table 5 were determined as intersections of the errors of the corresponding parameter for all five curves.

Owing to the considerably higher values of $\chi^{2}$ of our solutions compared with the corresponding critical level of $\chi_{0.001, n}^{2}$ (see the last rows of Table 5), we were unable to determine the "true" uncertainties of the fitted parameters that describe the disk, the hot line, and the spot. Instead we estimated the solution stability against the change of these parameters around their 

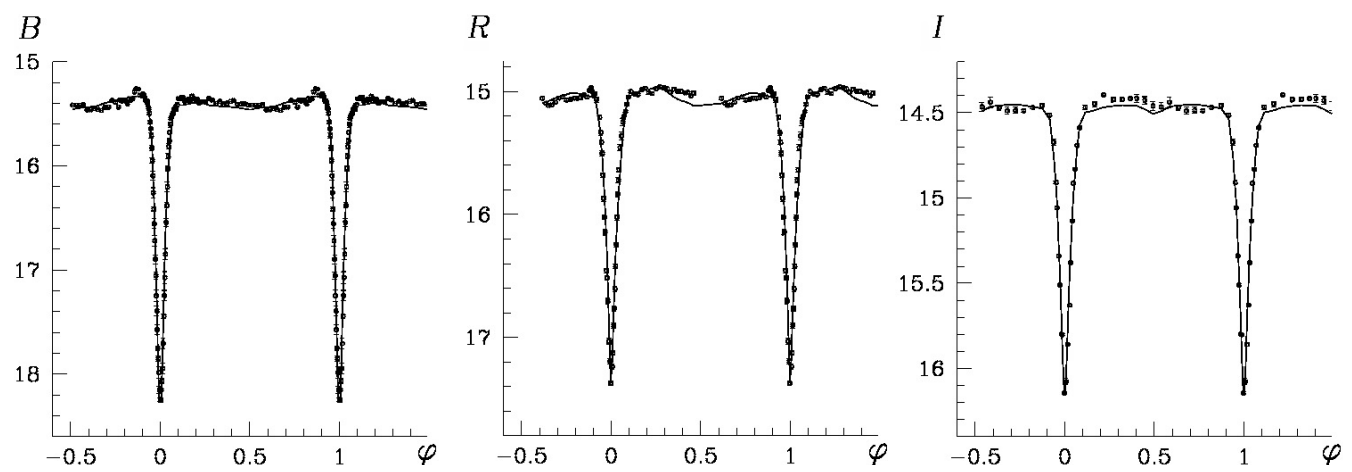

Fig. 6. Synthetic light curves (continuous lines) and observational data (points) in filters $B R I$.
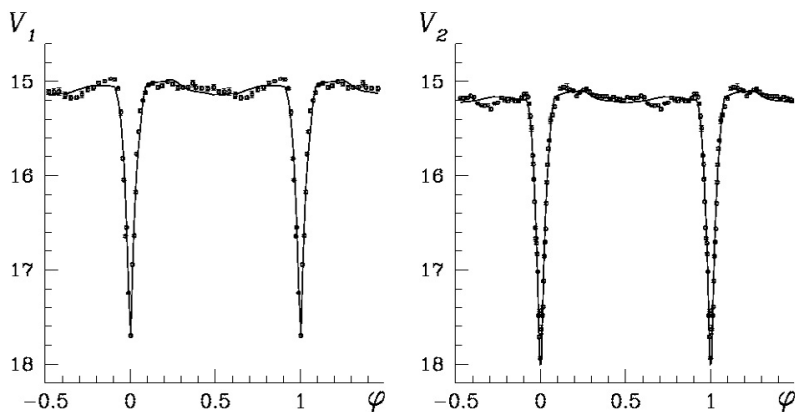

Fig. 7. Synthetic light curves (continuous lines) in filter $V$ and observational data (points) for the two observational seasons (January 2011 and December 2011).

derived values. Because there are too many fitted parameters in our problem, we simply varied each parameter $x_{k}$ in the range of its allowable values, while the remaining parameters were fixed at the values for which the lowest residual was obtained (corresponding solution). The highest deviation $\Delta x_{k}$ from the solution value $x_{k}$ for which the condition $\chi^{2}<\chi^{2}(+10 \%)$ was fulfilled was assumed as the uncertainty of the fitted parameter $x_{k}$ and is given in Table 5 .

Because the obtained ellipticity values $e$ of the accretion disk were close to zero for all light curves $(e \sim 0.00-0.02)$, the disk of J0107 could be assumed to be almost round. Then the effect of parameter $\alpha_{e}$ on the solution is negligible. For example, variations of $\alpha_{e}$ in the range from $0^{\circ}$ to $180^{\circ}$ for $e \sim 0.001$ lead to a change of $\chi^{2}$ up to $0.5-1 \%$.

Our solution means that the eclipse of the accretion disk is total only in filter $B$ (duration of the totality $\Delta \varphi_{B}=0.0134$ ) and partial in the remaining bands (filters).

The disk luminosity is considerably higher (see Fig. 8) than that of the remaining emission components (secondary star, white dwarf, and hot line). Accordingly, the eclipse depth and the light curve shape of J0107 mainly depend on the parameters of the accretion disk (Figs. 6-8). The white dwarf contributes very little to the total luminosity despite the high temperature because of its small radius. The hot line emission causes some features on the out-of-eclipse part of the light curves, which are more apparent in filters $V$ and $R$ (Fig. 8).

\subsection{Analysis of the global parameters}

The analysis of the obtained system parameters of J0107 (Table 5) led to several conclusions.

1. The obtained average radius of the secondary $\left\langle R_{2}\right\rangle=0.3181$ (in units $a_{0}$ ) is close to the average radius $R / a_{0}=0.3085$
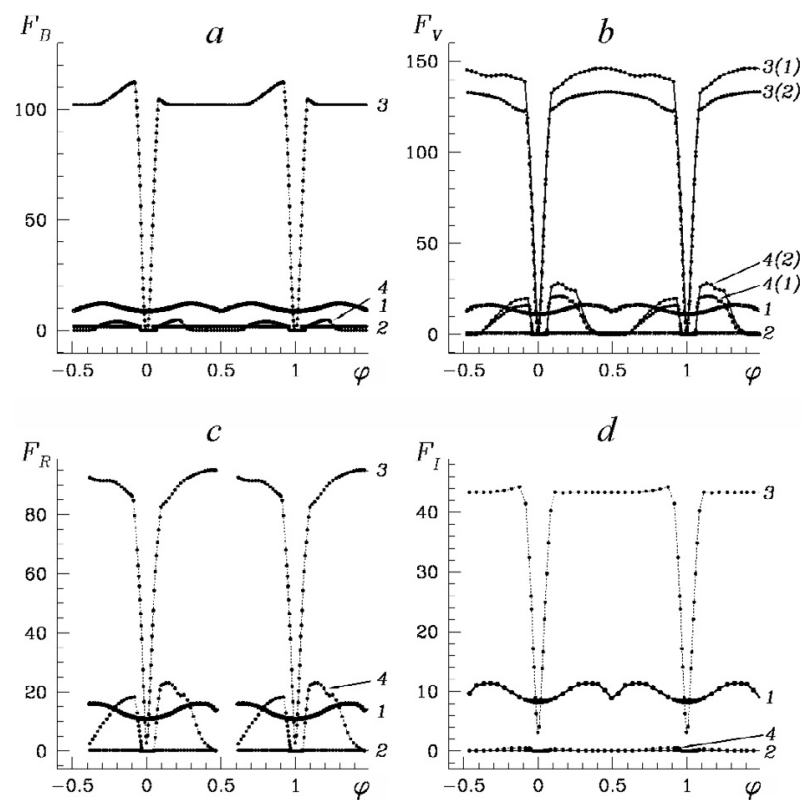

Fig. 8. Relative light contributions of the secondary (1), white dwarf (2), accretion disk with the hot spot (3) and hot-line (4) in filters $B(a)$, $V(b), R(c), I(d)$. These contributions are given separately for the two observational seasons (January 2011 and December 2011) in filter $V$.

of the star filling its Roche lobe obtained by the formulas of Eggleton (1983) for $q=2.332$.

2. The radius of the accretion disk is relatively small, comparable with that of the secondary component $\left(R_{\mathrm{d}} \sim 0.4 \xi\right)$. The disk size is smallest in filter $B$, which is the band of the deepest eclipse. The obtained value $\beta_{\mathrm{d}} \sim 5^{\circ}$ means that the disk of J0107 has a relatively thick outer edge. The huge luminosity and relatively small radius of the accretion disk mean relatively high temperature.

3. We were able to reproduce the photometric data of J0107 well with the values $q=M_{\mathrm{wd}} / M_{2}=2.332$ and $i=81.4^{\circ}$. However, the main contributor to the light curve of the system $\mathrm{J} 0107$ is the very bright accretion disk around the white dwarf, not the stellar components that define the value of $q$. Hence, radial velocity measurements of the target are necessary to obtain a more reliable value of the mass ratio $q$.

4. The obtained value $T_{\mathrm{wd}}=40000 \mathrm{~K}$ of $\mathrm{J} 0107$ is quite high. The comparison with the reliable measurements of $T_{\mathrm{wd}}$ in 43 CVs (Townsley \& Gänsicke 2009) reveals that only two of them, DW UMa and MV Lyr, have hotter white dwarfs (WDs) than J0107 and the $T_{\mathrm{wd}}$ of TT Ari is similar to that of J0107. These three cases (DW UMa, MV Lyr and TT Ari) are the only CVs of nova-like type from the whole sample. 


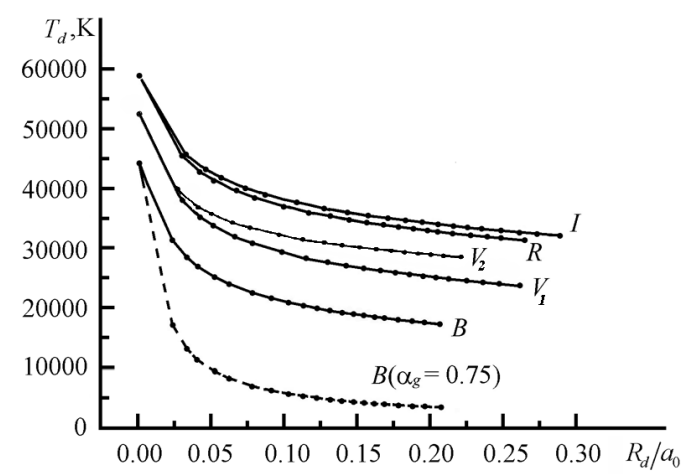

Fig. 9. Temperature distributions along the disk radius of J0107 for the different spectral bands. The dashed line shows the distribution for filter $B$ corresponding to the equilibrium value $\alpha_{g}=0.75$. The second point of each curve corresponds to the values $R_{\mathrm{in}}, T_{\mathrm{in}}$.

5. The temperature $T_{\text {in }}$ of the boundary layer of the disk exceeds $T_{\text {wd }}$ by factors of $1.1,1.31,1.46$, and 1.47 in filters $B$, $V, R$, and $I$. The $T_{\text {in }}$ values in all filters are well above the standard temperatures for cataclysmic variables at quiescence but are similar to those during their outbursts. The determined outer edge temperatures $T_{\text {out }}$ (Table 5) of J0107 are also considerably higher (by factors of 5-10) than the typical values $T_{\text {out }} \sim 3000-5000 \mathrm{~K}$ for the cataclysmic variables.

6. The obtained low values $0.16-0.27$ of $\alpha_{g}$ (Table 5) determine relatively flat temperature distributions along the disk radius (Fig. 9). These values are also typical for CVs during outbursts (when $\alpha_{g}$ decreases up to $\sim 0.1$ ).

The obtained temperatures of the white dwarf and accretion disk revealed that the high-temperature emission that heats the secondary is generated mainly by the boundary layer of the disk.

7. Special attention was paid to the obtained high temperature of $5090 \mathrm{~K}$ of the secondary of J0107. We attempted to obtain a good light curve solution for temperatures appropriate for a red dwarf secondary but they were far from the photometric data of $\mathrm{J} 0107$, especially in the $B$ band. The solutions for $T_{2}$ values from the range [3000, 4700] $\mathrm{K}$ corresponded to values of $\chi^{2}$ higher by $30-40 \%$ whereas the values of the main parameters $q, i, R_{\mathrm{wd}}$, and $T_{\mathrm{wd}}$ remained almost the same (within 1\%).

A review of Table 4 reveals that the high temperature of the secondary of J0107 is more similar to long-period SW Sex star. It was reasonable to assume that the high value of $T_{2}$ is caused by the heating through the emission of both the high-temperature white dwarf and the high-temperature accretion disk of $\mathrm{J} 0107$.

High temperatures of the secondaries of CVs have been established during their outbursts. For instance, Hessman et al. (1984) showed that during the eruption of the dwarf-nova SS Cyg the temperature of its K5 star increased to $16000 \mathrm{~K}$ on the side facing the white dwarf, while Robinson et al. (1986) found that this heating causes even an underestimation of the measured radial velocities. Obviously, the heating effect is essential for close binaries whose components have quite different temperatures, as our target.

Our detailed calculations of the reflection effect of J0107 revealed that it decreases the contribution of the ellipsoidal effect around phase $\varphi \sim 0.5$. Otherwise, the synthetic light curve of the secondary would have a deeper minimum at phase 0.5 than at phase 0.0 , and correspondingly, the total light curve of J0107 would have a dip around phase 0.5 . The very strong heating of the secondary in our case is the reason that the light flux at phase $\varphi \sim 0.5$ is comparable with the fluxes from the secondary at the quadratures. Indeed, the minimum of the secondary' light fluxes in the phase range $\varphi \sim 0.4-0.6$ (see Fig. 8) results from the covering (eclipse) of almost a quarter of the hottest surface of the secondary star by the accretion disk.

Our tests with temperatures $T_{\text {in }}$ lower than the value corresponding to our $V$ light curve solution (Table 5) led to a considerable worsening of the fit. For instance, $T_{\text {in }}=40000 \mathrm{~K}$ would cause a decrease of the secondary' $V$ flux of up to $12 \%$, while $T_{\text {in }}=20000 \mathrm{~K}$ would lead to a decrease of up to $18 \%$. Then the secondary' light curve would become the same as the curve without any heating. Hence, each underestimation of the secondary' heating causes undesired deviations of the synthetic light curves from the observational data.

Figure 10 shows the temperature distribution caused by the heating effect on the secondary' surface.

One could expect that the prolonged heating of the secondary surface in the part facing the white dwarf of J0107 would cause stationary streams in the photosphere that strive to equalize the temperatures of the neighboring volumes. Moreover, the turbulent processes (vortical and acoustic) in the photosphere of the late star (more intensive by two orders than in the hot star) also favor the establishment of thermal equilibrium. Although the energy transfer into the convective stellar layers is poorly studied, some rough estimation of its time scale can be obtained assuming that the thermalization of the absorbed heat occurs up to a depth of several photospheric thicknesses. This estimate shows that increasing the secondary surface temperature by $2000 \mathrm{~K}$ through the turbulent transfer with acoustic speed would take several days.

Consequently, the prolonged intensive heating from the hot white dwarf and from the hot accretion disk as well as the turbulent mixing of the photospheric gas of the secondary are probably the main reasons for the higher temperature $T_{2}$ of J0107 compared to those of the other nova-like variables with cooler white dwarfs.

8. The comparison of the light curve solutions of the $V$ light curves from December 2011 and January 2011 revealed a decrease of the disk radius, a decreasing thickness of the disk outer edge, an increasing temperature at the disk outer edge, and a flatter temperature distribution along the disk radius in December 2011 than that in January 2011 (Table 5).

\subsection{The non-steady emission of the accretion disk}

It is known that during the outbursts the emission of the CVs increases considerably (by factor of hundreds) due to the increased accretion. Moreover, the temperature of the CV disks increases and the temperature distribution along the disk radius becomes flatter (Djurašević 1996), i.e., the emission of the CVs at outbursts becomes non-steady.

Our light curve solution revealed that the parameter $\alpha_{g}$ of J0107 was $\sim 0.2$ for all filters, i.e., far from the steady state value 0.75 . Moreover, the empirical dependence of the emission of J0107 on the wavelength differs from the black-body type (Fig. 11), which is the second appearance of the non-steady emission of its accretion disk.

The theoretical fluxes in the red part of the spectrum ( $R$ and $I$ bands), which correspond to the light curve solution in filter $V\left(T_{\text {in }}=52468 \mathrm{~K}\right.$ and $\left.\alpha_{g}=0.2156\right)$, are half as strong as the observed fluxes (Fig. 11). Higher temperatures of the 

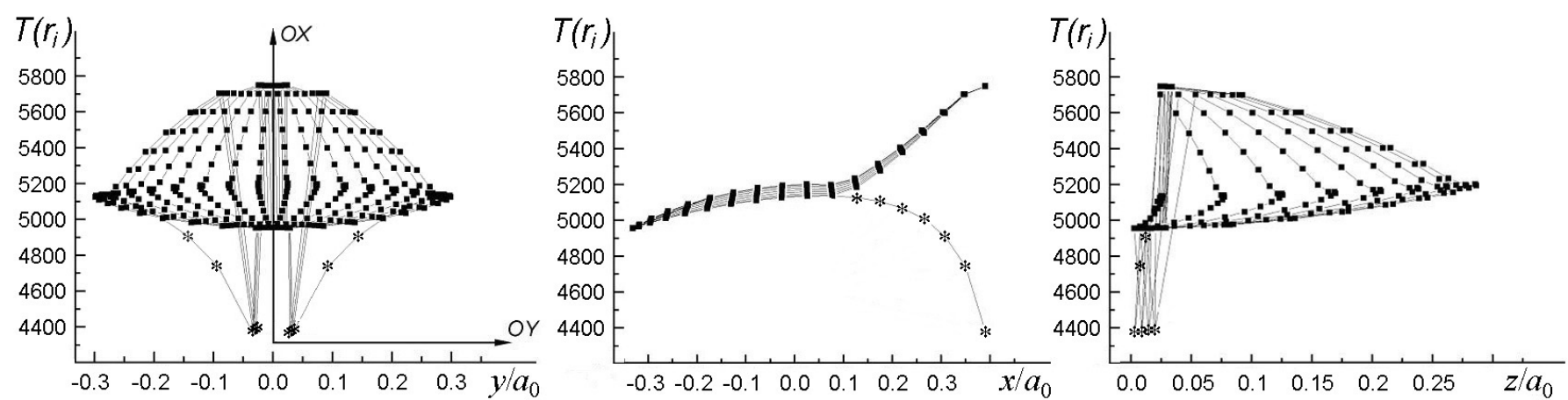

Fig. 10. Temperature distribution of the secondary surface corresponding to the parameters of J0107 from Table 5. The points near the orbital plane which are shielded by the disk edge are marked by asterisks.

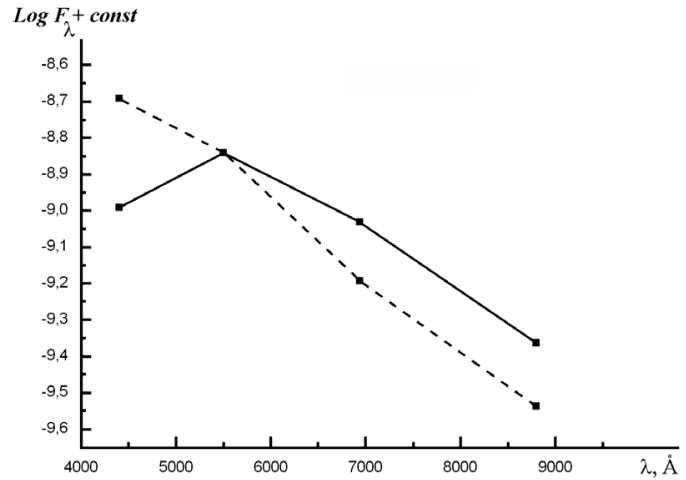

Fig. 11. Empirical dependence of the accretion disk emission on the wavelength (solid line) and the synthetic dependence of a disk with black-body emission whose parameters are equal to the solution for filter $V$ (dashed line). The points refer to the average fluxes at each filter for the phase range $0.2-0.7$ (without effects of disk eclipse and hot spot).

boundary layer $T_{\text {in }} \sim 58000-59000 \mathrm{~K}$ and a flatter temperature distribution along the disk $\left(\alpha_{g} \sim 0.16-0.17\right)$ than those in the $V$ filter were necessary to provide the observed disk emission flux and the relative contribution of the disk to the total system luminosity in the red part of the spectrum. A larger disk radius would not be able to give the necessary effect because it would lead to an eclipse shape different from the observed one. The situation for filter $B$ is the reverse: the observed disk flux is about two times smaller than the theoretical flux for the values of $T_{\text {in }}$ and $\alpha_{g}$ corresponding to the light curve solution in filter $V$ (Fig. 11).

With obtained value $T_{\mathrm{wd}}=40000 \mathrm{~K}$ and assuming an average mass $0.7 M_{\odot}$ of the white dwarf (see Table 4 ), we obtained an approximate estimation of the mass accretion rate of J0107 $\dot{M}=8 \times 10^{-9} M_{\odot} \mathrm{yr}^{-1}$ (according to formula (2) of Townsley \& Gänsicke 2009). This high value can explain the high luminosity of its disk.

The derived exceptionally high disk temperatures, the quite low values of the parameter $\alpha_{g}$, and the high mass accretion rate of J0107 are typical for CVs at eruption. But they are permanent for $\mathrm{J} 0107$ which means that its emission is permanently non-steady. We consider the similarities of the nova-like variable J0107 to CVs at eruption as additional arguments to the statement that the nova-like stars are permanently in an active state.

\subsection{The newly discovered nova-like variable J0107 versus the prototype UX UMa}

The empirical data and their analysis led us to the conclusion that the nova-like J0107 revealed many peculiarities. To exhibit them we compared the global characteristics of the newly discovered nova-like star J0107 with those of the well-known novalike eclipsing binary UX UMa whose photometry was fitted with the same model (Kjurkchieva et al. 2006; Khruzina et al. 2007). We found the following significant differences.

(a) The temperature of $40000 \mathrm{~K}$ of the white dwarf of J0107 is considerably higher than the corresponding value $27000 \mathrm{~K}$ of UX UMa.

(b) The temperature $T_{\text {in }}$ of the boundary layer of the accretion disk of J0107 is higher than $50000 \mathrm{~K}$, while that of UX UMa is below $30000 \mathrm{~K}$.

(c) The temperature of $5090 \mathrm{~K}$ of the secondary of J0107 is considerably higher than the corresponding value $3400 \mathrm{~K}$ of UX UMa.

(d) The characteristic temperatures of the hot line of J0107 are considerably higher (by a factor of 4-6) than those of the hot line of UX UMa.

We assume that these (mainly) temperature differences cause the different emission characteristics of the compared targets, which are:

(i) The empirical dependence of the emission on the wavelength of J0107 differs considerably from the black-body type, while that of UX UMa is almost of black-body type (see Fig. 16 in Kjurkchieva et al. 2006);

(ii) The $\alpha_{g}$ value of UX UMa is about 0.6, i.e., near to the steady-state value of 0.75 , while the $\alpha_{g}$ value of J0107 is about 0.2 , which is far from the equilibrium value.

Hence, there is considerable deviation of the emission of the bright accretion disk of J0107 from the steady type, while the emission of UX UMa is almost steady. We suggest that the nonsteady emission of the accretion disk of J0107 may be attributed to the low viscosity of the disk matter caused by its unusual high temperature. Analyses of the emission of more targets of this type are necessary to check this assumption.

An additional difference between J0107 and UX UMa is that the $\mathrm{H} \alpha$ emission line of UX UMa has a two-peaked profile at most phases, while that of J0107 is rather single-peaked.

\subsection{SW Sex classification of J0107}

The inherent features of SW Sex stars continuously evolve with increasing statistics but the same is true for the other subtypes of CVs. Moreover, some characteristics of the different subtypes overlap. This means that the rich world of CVs does not permit firm boundaries between the various types.

In order to check if the new photometric and spectral data and their modeling support or disclaim the initial supposition 
about the belonging of J0107 to the SW Sex class we used the characteristics of this subtype as defined by Thorstensen et al. (1991), Rodríguez-Gil et al. (2007), and Schmidtobreick et al. (2009).

(a) J0107 shows the typical V-shaped eclipse of SW Sex stars, maybe one of the first main signs of this subtype. Recently, an eclipse is not considered a mandatory criterion of the SW Sex stars because it is biased toward high-inclination systems. Today, at least 13 out of a total of 35 confirmed SW Sex stars do not display eclipses (Rodríguez-Gil et al. 2007).

(b) Our target poses high-velocity emission line wings, typical of SW Sex stars (Table 3).

(c) The one-peaked profile of the $\mathrm{H} \alpha$ emission line is typical of SW Sex stars (Thorstensen et al. 1991).

(d) J0107 shows a very weak He I 6678 emission line, which is typical of SW Sex stars.

(e) Our low-resolution spectra revealed an S-wave for the $\mathrm{H} \alpha$ line with an amplitude of about $270 \mathrm{~km} \mathrm{~s}^{-1}$ (Table 3).

(f) J0107 shows high luminosity and a hot white dwarf, typical of SW Sex subtype.

(g) The obtained high value $8 \times 10^{-9} M_{\odot} \mathrm{yr}^{-1}$ of the mass accretion rate of $\mathrm{J} 0107$ is another confirmation that it belongs to the SW Sex stars.

Owing to the low-resolution of our spectra, their insufficient phase coverage, and insufficient $\lambda$ coverage, we are unable to confirm (or disprove) the additional spectral signs of SW Sex stars (Rodríguez-Gil et al. 2007): narrow absorption features in the Balmer and He I lines near the inferior conjunction of the white dwarf; orbital phase offsets of the radial velocity curves with respect to the photometric ephemeris; He II 4686 emission; line flaring (change of the emission line flux and velocity with periods of about 10-20 min) similar to that in the intermediate polar CVs.

The value $P_{\text {orb }}=4.65 \mathrm{~h}$ of $\mathrm{J} 0107$ is slightly higher than the period range 3.0-4.5 h of the most of the known SW Sex stars (Rodríguez-Gil et al. 2007). But there are members with considerably longer periods (see our Table 3 and Table 6 of Rodríguez-Gil et al. 2007).

It was established recently that half of the nova-likes known to undergo VY Scl faint states (system brightness drops by up to 4-5 mag for months) are SW Sex stars (Rodríguez-Gil et al. 2007). Until today, J0107 has not shown VY Scl-type light decreasing.

According to Schmidtobreick et al. (2009) an S-wave and central absorption are the main characteristics of the SW Sex stars, and correspondingly, they should be searched for with spectral observations. Reviewing the available information for the SW Sex variables, we established that especially important characteristics of these stars are the high temperatures of their white dwarfs and accretion disks. To exhibit these, photometric data are necessary (also in UV and X-ray regions).

\subsection{Evolution state}

After establishing that the most of the nova-like stars in the 3-4 h period range (just above the period gap) are of SW Sex subtype (Rodríguez-Gil et al. 2007; Schmidtobreick et al. 2009), it was reasonable to assume that because of the angular momentum loss each CV with a longer orbital period will fall into the 3-4 h period range and will turn into a SW Sex star, i.e., the SW Sex phenomenon can be considered as an evolutionary stage of the CV population.
The important evolution role of the SW Sex stars requires future precise observations and modeling to study and to explain their peculiarities.

The unusual high luminosities of the SW Sex stars are not properly explained. High mass-transfer (Livio \& Pringle 1994) or an additional light source (such as nuclear burning, Honeycutt 2001) were proposed as plausible reasons.

Recently, it was assumed that the accretion at a very high rate $\dot{M}$ is the most likely explanation for the high luminosity of the SW Sex stars. It may be caused by enhanced mass transfer from the donor star due to heating of its inner face by a very hot white dwarf. Indeed, just the nova-like CVs have the hottest white dwarfs (with effective temperatures of up to $50000 \mathrm{~K}$ ) found in any CVs (Townsley \& Gänsicke 2009). The results of our light curve solution of J0107 completely confirm all previous theoretical suggestions.

The extremely high accretion rates of the SW Sex stars (around $5 \times 10^{-9} M_{\odot} \mathrm{yr}^{-1}$, Townsley \& Bildsten 2003) explain their permanent high state but why SW Sex stars have the highest mass-transfer rates it has yet to be satisfactorily explained. This is a cornerstone because it is obvious that the mass-transfer rates govern the evolution of CVs.

The high values of $T_{\text {wd }}$ and $\dot{M}$ are assumed to be common characteristics of VY Scl and SW Sex stars. It was suggested that these two subtypes represent a phase in the CV evolution at which the binaries evolve into semi-detached configurations with a short peak of the mass transfer (Rodríguez-Gil et al. 2007). Some SW Sex stars do undergo VY Scl-type light decreasing (for instance DW UMa, classified even as VY Scl subtype by Townsley \& Gänsicke 2009), but there are dwarf novae (like HT Cas and RX And, Schreiber et al. 2002) that also show VY Scl-type light decreasing. New data and analyses are necessary to derive additional criteria for the sub-classification of the CVs and to arrange them chronologically in the evolution scheme.

\section{Conclusions}

The light curve solution of the nova-like variable 2MASS J01074282+4845188 led to the following conclusions.

1. The observed excessively deep light minimum is reproduced by the eclipse of the very bright accretion disk, whose contribution is much higher than those of the other light sources.

2. The obtained unusual high temperatures of the disk of J0107 and the low values of the parameter $\alpha_{g}$ are typical of Cvs during outburst. These similarities of our new nova-like variable and the CVs at eruption might be additional arguments for the statement that the nova-like stars are permanently in an active state.

3. The flat temperature distribution along the disk radius and the deviation of the energy distribution from the black-body law mean that the disk emission of J0107 is non-steady. This result can be attributed to the low viscosity of the disk matter due to its unusual high temperature.

4. The primary of J0107 is one of the hottest white dwarfs in CVs.

5. The temperature $5090 \mathrm{~K}$ of the secondary of J0107 is quite high and more appropriate to a long-period SW Sex star. Our detailed analysis revealed that it could be mainly attributed to the intense heating from the hot white dwarf and the hot accretion disk.

6. The high mass accretion rate $\dot{M}=8 \times 10^{-9} M_{\odot} \mathrm{yr}^{-1}$ of $\mathrm{J} 0107$ implies that it is in a very short evolutionary stage. 
7. The broad and single-peaked $\mathrm{H} \alpha$ profile of J0107 and the S-wave surely confirm its classification as an SW Sex star.

8. The main differences between the global parameters of J0107 and those of the well-known nova-like eclipsing binary UX UMa are the considerably higher temperatures of all components of the J0107 configuration (white dwarf, accretion disk, secondary, and hot line) compared to those of UX UMa.

Initially, the newly discovered nova-like eclipsing star J0107 attracted our attention because of its excessively deep eclipse. Furthermore, it turned out that this is the deepest permanent eclipse (at least during 11 months) among the known nova-like stars. Finally, the light curve solution showed that some global parameters of J0107 have quite extreme values (especially the temperatures). We conclude that the established peculiarities of 2MASS J01074282+4845188 make it an interesting target for follow-up spectral observations and observations in UV and $\mathrm{X}$-ray bands.

Acknowledgements. This study is supported by funds of the projects DO 02 362, DO 02-85 and DDVU 02/40-2010 of the Bulgarian National Science Fund, as well as by the Russian Foundation for Basic Research (project nos. 09-0200225, 11-02-00258) and the Program of State Support to Leading Scientific Schools of the Russian Federation (NSh-2374.2012.2). D.D. and D.K. gratefully acknowledge observing grant support from the Institute of Astronomy and Rozhen National Astronomical Observatory, Bulgarian Academy of Sciences. The authors are very grateful to the anonymous referee for the useful recommendations and to K.V. Bychkov from the Moscow University for the discussion about the time-scale of heating of the secondary star. This research makes use of the SIMBAD and Vizier data bases, operated at CDS, Strasbourg, France, and NASA Astrophysics Data System Abstract Service.

\section{References}

Abubekerov, M. K., Gostev, N. Y., \& Cherepashchuk, A. M. 2008, Astron. Rep., 52,99

Al-Naimiy, H. M. 1978, Ap\&SS, 53, 181

Balog, N. I., Goncharskij, A. V., \& Cherepashchuk, A. M. 1981, Sov. Astron., 25,38

Basko, M. M., \& Sunyaev, R. A. 1973, Ap\&SS, 23, 71

Bisikalo, D. V., Boyarchuk, A. A., Kuznetsov, O. V., \& Chechyotkin, V. M. 1997, Astron. Rep., 41, 786
Bisikalo, D. V., Boyarchuk, A. A., Chechetkin, V. M., Kuznetsov, O. A., \& Molteni, D. 1998, MNRAS, 300, 39

Bisikalo, D. V., Boyarchuk, A. A., Kaigorodov, P. V., \& Kuznetsov, O. A. 2003, Astron. Rep., 47, 809

Bisikalo, D. V., Kaigorodov, P. V., Boyarchuk, A. A., \& Kuznetsov, O. A. 2005 Astron. Rep., 49, 701

Cherepashchuk, A. M. 1993, AZh, 70, 1157

Cherepashchuk, A. M., Katysheva, N. A., Khruzina, T. S., \& Shugarov, S. Y. 1996, in Highly evolved close binary stars (Amsterdam: Gordon and Breach), 1

Devor, J., Charbonneau, D., O’Donovan, F. T., Mandushev, G., \& Torres, G. 2008, AJ, 135, 850

Dimitrov, D. P., \& Kjurkchieva, D. P. 2012, New Astron., 17, 34

Djurašević, G. 1996, Ap\&SS, 240, 317

Eggleton, P. P. 1983, ApJ, 268, 368

Grygar, J., Cooper, M. L., \& Jurkevich, I. 1972, Bull. Astron. Inst. Czechosl., 23,147

Hessman, F. V., Robinson, E. L., Nather, R. E., \& Zhang, E.-H. 1984, ApJ, 286, 747

Himmelblau, D. 1972, Applied nonlinear programming (McGraw-Hill)

Honeycutt, R. K. 2001, PASP, 113, 473

Johnson, H. L. 1965, ApJ, 141, 923

Khruzina, T. S. 2000, Astron. Rep., 44, 446

Khruzina, T. S. 2001, Astron. Rep., 45, 255

Khruzina, T. S. 2011, Astron. Rep., 55, 425

Khruzina, T. S., Cherepashchuk, A. M., Bisikalo, D. V., Boyarchuk, A. A., \& Kuznetsov, O. A. 2003, Astron. Rep., 47, 214

Khruzina, T. S., Kjurkchieva, D., Marchev, D., \& Djurasevic', G. 2007, Astron. Rep., 51, 318

Kjurkchieva, D., Marchev, D., Khruzina, T., \& Djurašević, G. 2006, Ap\&SS, 306, 217

Littlefair, S. P., Dhillon, V. S., Marsh, T. R., et al. 2008, MNRAS, 388, 1582

Livio, M., \& Pringle, J. E. 1994, ApJ, 427, 956

Papadaki, C., Boffin, H. M. J., Stanishev, V., et al. 2009, J. Astron. Data, 15, 1

Ritter, H., \& Kolb, U. 2003, A\&A, 404, 301

Robinson, E. L., Zhang, E.-H., \& Stover, R. J. 1986, ApJ, 305, 732

Rodríguez-Gil, P., Gänsicke, B. T., Hagen, H.-J., et al. 2007, MNRAS, 377, 1747

Schmidtobreick, L., Rodríguez-Gil, P., \& Gänsicke, B. 2009, in Rev. Mex. Astron. Astrofis. Conf. Ser., 35, 115

Schreiber, M. R., Gänsicke, B. T., \& Mattei, J. A. 2002, A\&A, 384, L6

Shakura, N. I., \& Sunyaev, R. A. 1973, A\&A, 24, 337

Stanishev, V., Kraicheva, Z., Boffin, H. M. J., et al. 2004, A\&A, 416, 1057

Thorstensen, J. R., Ringwald, F. A., Wade, R. A., Schmidt, G. D., \& Norsworthy, J. E. 1991, AJ, 102, 272

Townsley, D. M., \& Bildsten, L. 2003, ApJ, 596, L227

Townsley, D. M., \& Gänsicke, B. T. 2009, ApJ, 693, 1007

Warner, B. 1995, Ap\&SS, 232, 89 